\title{
Cholesteric liquid crystal paints: in situ photopolymerization of helicoidally stacked multilayer nanostructures for flexible broadband mirrors
}

\author{
Dae-Yoon Kim', Kyung Min Lee ${ }^{2}$, Timothy J. White ${ }^{2,3}$ and Kwang-Un Jeong ${ }^{4}$
}

\begin{abstract}
Utilizing sequential layer-by-layer (LBL) coating and subsequent photopolymerization, we developed circularpolarizing and free-standing mirrors with a broad reflection bandwidth. Photopolymerizable cholesteric liquid crystal (CLC) paints were first prepared by considering the intrinsic chirality and the coating viscoelasticity. The polymerstabilized nanostructures had finely tuned helical pitches that selectively reflected light over the entire visible wavelength range. The CLC films exhibited excellent thermomechanical properties and chemical stabilities, which enabled the preparation of patterned optical objects at macroscopic dimensions. The LBL coating and photopolymerization of the CLC paints demonstrated here can provide a simple solution for manufacturing flexible photonic devices with large areas at low cost.
\end{abstract}

\section{Introduction}

The structural color and light control of photonic crystals have been the subject of intensive research ${ }^{1}$. Because incident light encounters multiple thin layers or helicoidally stacked nanostructures in photonic crystals, it is strongly reflected by the constructive interference between the reflections from the array of transparent materials ${ }^{2}$. When the periodic modulation due to the alternating high and low refractive indices is proportional to the visible wavelength region, the partial photonic bandgap is opened ${ }^{3}$. This light is completely reflected in the photonic crystal, and a selective wavelength with an iridescent color is observed ${ }^{4}$. Grubbs et al. ${ }^{5}$ prepared highly ordered reflectors via the nanophase separation of block copolymers. Thomas et al. ${ }^{6}$ fabricated color-tunable

Correspondence: Kwang-Un Jeong (kujeong@jbnu.ac.kr)

${ }^{1}$ Department of Materials Science and Engineering, Massachusetts Institute of Technology, Cambridge, MA 02139, USA

2US Air Force Research Laboratory, Wright-Patterson Air Force Base, Dayton, $\mathrm{OH}$ 45433, USA

Full list of author information is available at the end of the article. films by manipulating the face-centered cubic structure of nanoparticles. Self-organized periodic structures found in nature inspire the design and synthesis of new photonic elements ${ }^{7}$.

In this research, a cholesteric liquid crystal (CLC) that is paintable and polymerizable is used to prepare a flexible broadband mirror ${ }^{8,9}$. The CLC is a chiral version of a nematic mesophase ${ }^{10}$. It exhibits a Bragg reflection with the relationship $\lambda_{0}=n \times P_{0}$, where $\lambda_{0}=$ notch position, $n=$ average refractive index, and $P_{0}=$ pitch length. The bandwidth $(\Delta \lambda)$ of the CLC is determined by the birefringence of the LC and the length of the helical pitch $(P)$, $\Delta \lambda=\Delta n \times P$. The birefringence $(\Delta n)$ is defined as $\left(n_{\mathrm{e}}-n_{\mathrm{o}}\right)$, where $n_{\mathrm{e}}$ and $n_{\mathrm{o}}$ are the extraordinary and ordinary refractive indices, respectively. Due to the helicoidal superstructure, the selective reflection of the CLC is circularly polarized ${ }^{11}$. When unpolarized light is incident on a CLC, a maximum of $50 \%$ of the light is reflected (matching the circular handedness), and $50 \%$ of the light is transmitted.

\section{(c) The Author(s) 2018}

(c) (i) Open Access This article is licensed under a Creative Commons Attribution 4.0 International License, which permits use, sharing, adaptation, distribution and reproduction cc) in any medium or format, as long as you give appropriate credit to the original author(s) and the source, provide a link to the Creative Commons license, and indicate if changes were made. The images or other third party material in this article are included in the article's Creative Commons license, unless indicated otherwise in a credit line to the material. If material is not included in the article's Creative Commons license and your intended use is not permitted by statutory regulation or exceeds the permitted use, you will need to obtain permission directly from the copyright holder. To view a copy of this license, visit http://creativecommons.org/licenses/by/4.0/. 
This unique optical activity is derived from a helicoidally self-assembled feature of the CLC ${ }^{12}$. Generally, the addition of a chiral dopant to an achiral LC medium produces a helical nanostructure ${ }^{13}$. Because this method for achieving photonic materials is simple, many studies have focused on manipulating the reflection bandgap ${ }^{14}$. Earlier efforts to achieve broadband reflectors involved physically overlapping individual optical cells filled with CLCs with different $P$ values ${ }^{15,16}$. Because CLCs respond to external stimuli, a broadband reflector can be fabricated by thermal diffusion to obtain a pitch gradient ${ }^{17,18}$. In some cases, a CLC containing a light-driven molecular switch is utilized because the helical twisting power (HTP) can be gradually changed along the film thickness direction by a noncontact method $^{19}$. However, this method requires a large number of optical cells, and the device is difficult to manufacture due to its weight and the complexity of the electrical $\operatorname{design}^{20}$. Additionally, this type of low-molecular-weight system is disadvantageous because it cannot withstand a certain degree of mechanical deformation due to a lack of sufficient structural integrity ${ }^{21}$.

When photonic materials with free-standing character can be obtained, they can open routes to new applications, such as flexible devices ${ }^{22,23}$. In this respect, cholesteric polymers have been widely discussed because they exhibit both the orientational order of LC molecules and the elastic behaviors of polymeric networks ${ }^{24}$. Most studies of cholesteric polymers involve ultraviolet (UV) lightinduced polymerization ${ }^{25}$. Broer et al. ${ }^{26}$ reported broadband reflectors with a distribution of $P$ values, which arose due to the presence of asymmetric polymer networks that extended from the top to the bottom of the optical cell. However, another series of work was based on the diffusion-controlled phase separation of a reactive mesogen $(\mathrm{RM})^{27}$. Thus, the polymerization rate should be precisely engineered to achieve the optimal effects by considering the intensity of the UV light, the concentration of the absorption dye, and the thickness of the substrate gap $^{28}$.

Here the ability to fabricate flexible broadband mirrors by directly coating CLC paints consisting of chiral and achiral RM mixtures is explored. These new photonic materials exhibit excellent coating properties for both hard and flexible substrates that are flat and curved. The helical axis (HA) of the CLC paint is controlled by applying a shear force with a brush and applicator, which enables the discovery of brilliant reflection color in the visible range. Fast in situ photopolymerization of the CLC paints then freezes the helicoidal arrangement of the anisotropic molecules. Compared to the initial monomer state of the CLC paint, the thermomechanical stability of the polymer form in the crosslinked CLC film is significantly higher. Additionally, because phase separation is suppressed by the identical reactivities in the completely polymerizable system, the CLC film exhibits the same selective reflection of light as the CLC paint. In this work, the free-standing character and pitch-broadening of the CLC films are demonstrated. By applying continuous layer-by-layer (LBL) coatings of the CLC paints and subsequently photopolymerizing them in situ, three layers of red, green, and blue CLC films can be vertically stacked without the need for high-temperature curing for layer alignment, a mixture-filling process in the optical cell, and an adhesive lamination method for obtaining a single heterostructure.

\section{Materials and methods}

A chiral RM (cRM) with a helical twisting power of $22 \mu \mathrm{m}^{-1}$ was synthesized according to a previously reported method ${ }^{29}$. The achiral RMs $\mathrm{mRM}$ and $\mathrm{dRM}$ were used as received without further purification. Irgacure 651 was used as the photoinitiator. The cRM, mRM, and dRM were homogeneously blended at $80^{\circ} \mathrm{C}$ using a vortex mixer (WiseMix VM10) to give the CLC paints. The CLC films were prepared by performing a bar-coating (Elcometer 3580) method on bare glass at $25^{\circ} \mathrm{C}$. The thickness of CLC films was controlled to $10 \mu \mathrm{m}$ by changing the height of the casting knife. The thickness was measured using digital micrometer calipers (Mitutoyo Absolute). For the photopolymerization, the samples were irradiated with an UV source (Ushio SP9) with a light intensity of $20 \mathrm{~mW} \mathrm{~cm}{ }^{-2}$ for $10 \mathrm{~min}$.

\section{Experimental procedures}

The optical textures of the cholesteric mesophases were studied by polarized optical microscopy (POM, Nikon Eclipse E600). The transmittance spectra were detected by an UV and visible spectrophotometer (Jasco Arsn733) to measure the reflection property. The conversion rate from the CLC paints to the CLC films was evaluated by Fourier transform infrared spectroscopy (FTIR, Jasco 300E). The thermal behaviors were determined by thermogravimetric analysis (TGA, TA Q50) and differential scanning calorimetry (DSC, TA Q20) at a heating rate of $10^{\circ} \mathrm{C} \mathrm{min}^{-1}$. The storage modulus $\left(E^{\prime}\right)$ and loss tangent $(\tan \delta)$ of the CLC films were measured by dynamic mechanical analysis (TA Q800) with operating cantilevers of $10 \mathrm{~mm} \times 5 \mathrm{~mm} \times 10 \mu \mathrm{m}$. The mechanical stability was analyzed by a field adhesion test (ASTM D3359) and pencil-scratch test (ASTM D3363). One-dimensional (1D) wide-angle X-ray diffraction (WAXD) experiments were conducted with a Rigaku generator $(\mathrm{Cu} \mathrm{K \alpha} 12 \mathrm{~kW})$. The diffraction peak positions were calibrated with silicon crystals. For the scanning electron microscopy (SEM, Carl Zeiss 40VP) observations, the CLC films were completely frozen in liquid nitrogen for $15 \mathrm{~min}$ and then rapidly cut with a razor blade. The macroscopic photographs were obtained using a digital camera (Canon EOS 5D). 
(a)

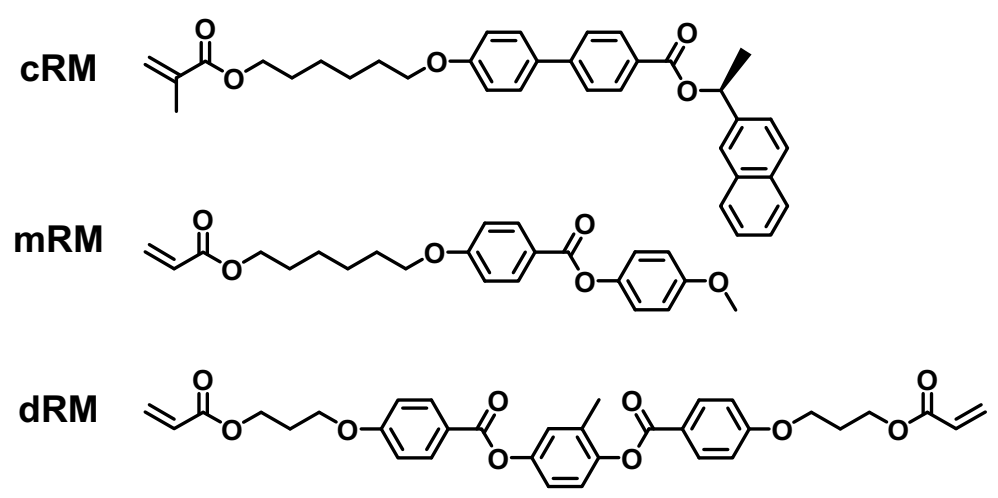

(b)
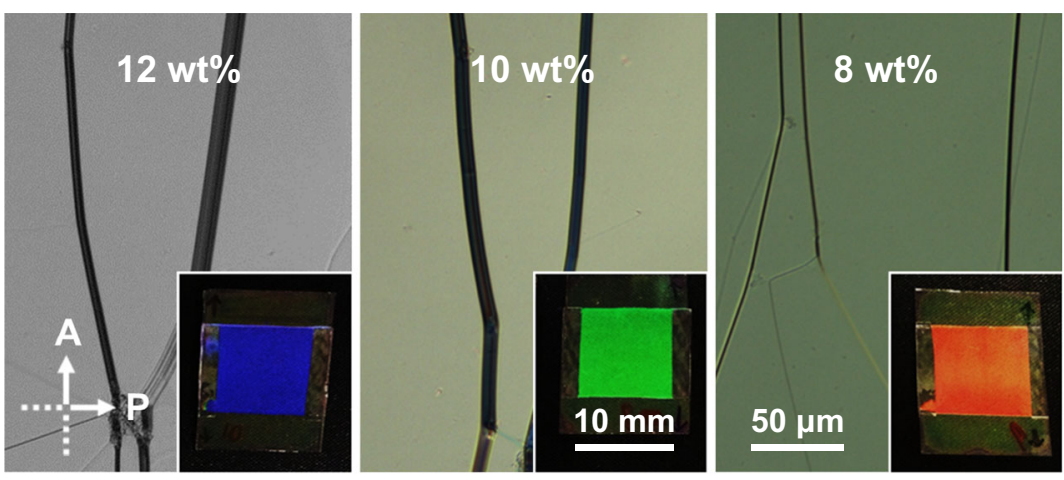

Fig. 1 Chemical structures of the chiral and achiral RMs used to construct the helical nanostructures (a). POM images of the CLC paints and their corresponding real cell images (insets) at room temperature (b)

\section{Results}

To achieve broadband photonic elements, CLC paints are first prepared using an optimized composition for coating. A cRM with a photopolymerizable methacrylate group is a core component of the CLC paints, as shown in Fig. 1a. Due to the presence of an $R$-configured naphthyl group, simply adjusting the concentration of the cRM in the nematic liquid crystal (NLC) medium enables the formation of cholesteric mesophases. In this study, a monoacrylate monomer (mRM) and a diacrylate monomer (dRM) are purposely used not only as the NLC host but also as chemical crosslinkers ${ }^{30}$. Based on the HTP of the CRM, the CLC paints are formulated with CRM:mRM: $\mathrm{dRM}$ weight percent ratios of 12:25:63, 10:25:65, and 8:25:67. Although the cholesteric mesophase of the CLC paints is only composed of the cRM and dRM, the mRM is also added to prevent the crystallization of the mixture (Fig. 1b). For the CLC paints obtained with the CRM and $\mathrm{dRM}$, the growth of a needle-like texture is observed in the POM microphotographs after a few minutes (Figure S1 in the Supporting Information). However, the CLC paints with the mRM maintain their ordered structure without any phase separation, which results in their dramatically enhanced processability, even at the room temperature (Figure S2 in the Supporting Information). In the planar anchoring condition, the molecules lie down on the surface and the HA of self-assembled helical nanostructure is parallel to the surface normal. Therefore, the mRM and dRM mixtures doped with 12, 10, and $8 \mathrm{wt} \%$ cRM clearly exhibit the selective reflection of blue, green, and red light, respectively (insets in Fig. 1b).

CLC paints with unique optical properties and a suitable viscoelasticity can be used for various applications, such as architectural coatings, colorimetric sensors, and information displays ${ }^{31}$. In this work, the CLC paints are applied to artificial nails, evenly brushed in one direction and then irradiated with UV light to stabilize the ordered structure through the formation of a polymer network. As shown in Fig. 2a, the pinky, ring, and middle fingers covered with the CLC films are red, green, and blue, respectively. The ability to localize the presence and color of the CLC paints is further described. The artificial nails on the thumb and index fingers illustrate prototypical symbols of the cholesteric character. When the CLC paints described above are cast on a glass substrate, a freestanding and circular-polarizing photonic material with macroscopic dimensions can be fabricated. The cast sample is evenly sheared with a bar coater. Then, the in situ photopolymerization of the coated sample is performed to obtain a robust optical film. The photoinitiated polymerization is induced by exposure to $365 \mathrm{~nm}$ UV light for $10 \mathrm{~min}$. Based on a field adhesion method (ASTM 

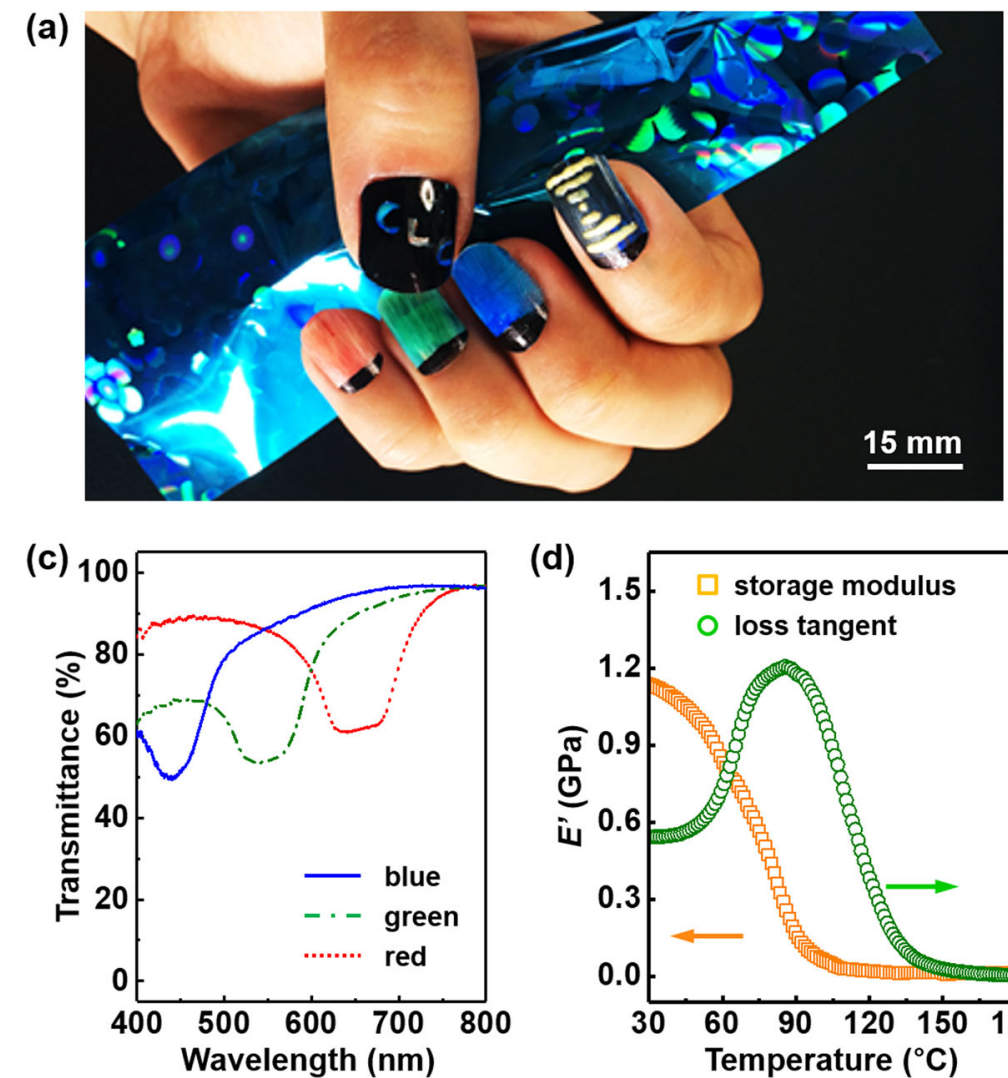

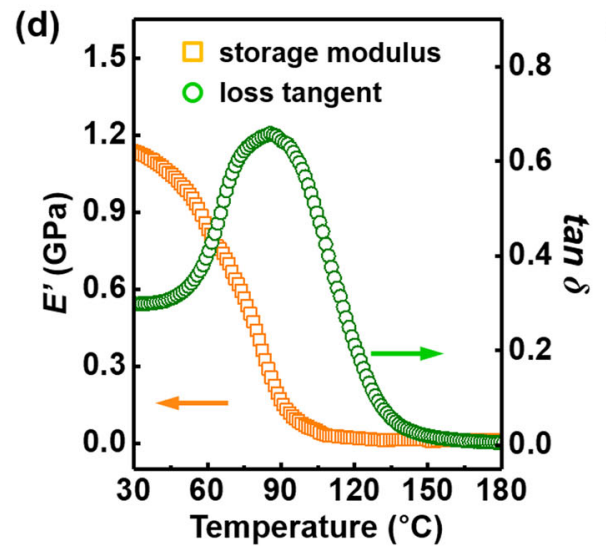

(b)

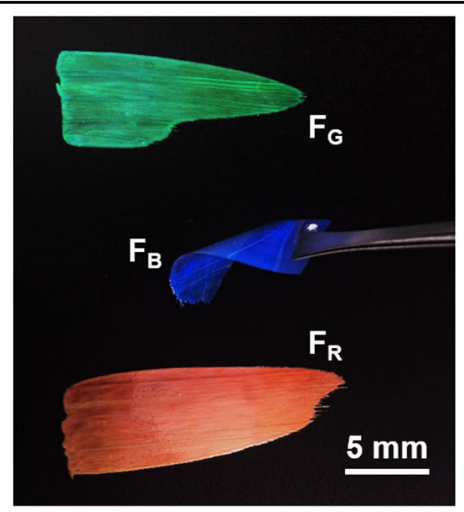

(e)

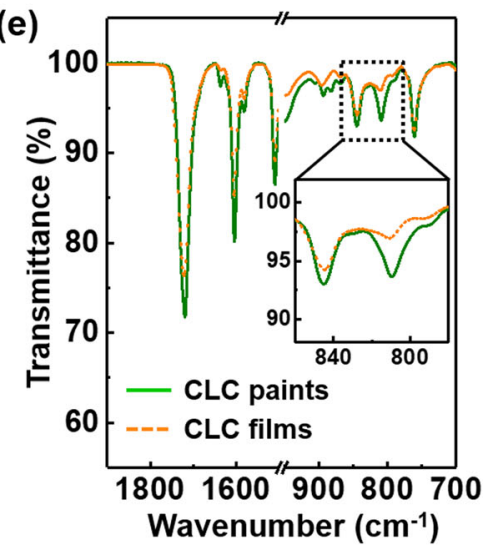

Fig. 2 Application of the chirophotonic crystal paints as fingernail polish (a). Macroscopic images of three free-standing CLC films illustrating the iridescence inherent to the cholesteric mesophase and the retention of the blue, green, and red reflection colors (b). Transmittance spectra indicating that the photopolymerized CLC paints reflect red, green, and blue (c). DMA thermograms of $F_{B}(\mathbf{d})$. Transformation of the CLC paints to films after UV polymerization (e)

D3359), the CLC films exhibit better mechanical properties than the CLC paints (Figure S3 in the Supporting Information) $)^{32}$. For the CLC paints, approximately $80 \%$ of the crosshatch pattern is removed by the tape peel action. The adhesion grade of the CLC paints on the glass substrate is estimated to be $0 \mathrm{~A}$. However, the crosshatch zone is undamaged for the CLC films on plastic (polyethylene terephthalate), glass (bare substrate), and metal (stainless steel) substrates. After the tape peel action, no debris is detected on the tested tape side. Therefore, the CLC films can be rated $4 \mathrm{~A}$ in terms of the adhesion strength. It is possible to fabricate the optically active layer directly on the substrate, which provides flexibility for designing optoelectronic devices.

The polymeric stabilization of the CLC paints can be easily handled with a tweezer (Fig. 2b). The thickness of the CLC films is controlled to $10 \mu \mathrm{m}$. The colors of the CLC films observed by the naked eye are nearly the same as those of the CLC paints. The proposed fully reactive cholesteric system is intentionally designed to minimize phase separation during photopolymerization ${ }^{33}$. The acrylate-functionalized chiral and achiral molecules exhibit identical reactivities. Therefore, the fast photopolymerization freezes the helical nanostructure. The photonic materials fabricated with 12,10 , and $8 \mathrm{wt} \% \mathrm{cRM}$ have red $\left(\mathrm{F}_{\mathrm{R}}\right)$, green $\left(\mathrm{F}_{\mathrm{G}}\right)$, and blue $\left(\mathrm{F}_{\mathrm{B}}\right)$ colors, respectively. The corresponding characteristic light reflections are $\lambda_{\max }=435,525$, and $630 \mathrm{~nm}$, respectively (Fig. 2c). These results also indicate good dimensional stability. The nearly identical positions of the reflection notches of the CLC paints and films indicate that the $P$ value is not affected by the photopolymerization (Figure S4 in the Supporting Information). The thermomechanical properties of the CLC films are examined by a dynamic mechanical analyzer (DMA). In the example at the top, the $F_{B}$ sample exhibits good flexibility. It has a glass transition temperature $\left(T_{\mathrm{g}}\right)$ of $85^{\circ} \mathrm{C}$ (obtained from the peak value of the $\tan \delta$ curve) and a storage modulus $\left(E^{\prime}\right)$ of $1.2 \mathrm{GPa}$ at room temperature (Fig. $2 \mathrm{~d}$ ). The thermomechanical properties determined by DMA are in good agreement with those measured by DSC, which confirms that $T_{\mathrm{g}}=85^{\circ} \mathrm{C}$ (Figure S5 in the Supporting Information). Under these conditions, the conversion of the chiral and achiral RMs is calculated by monitoring the change in the 

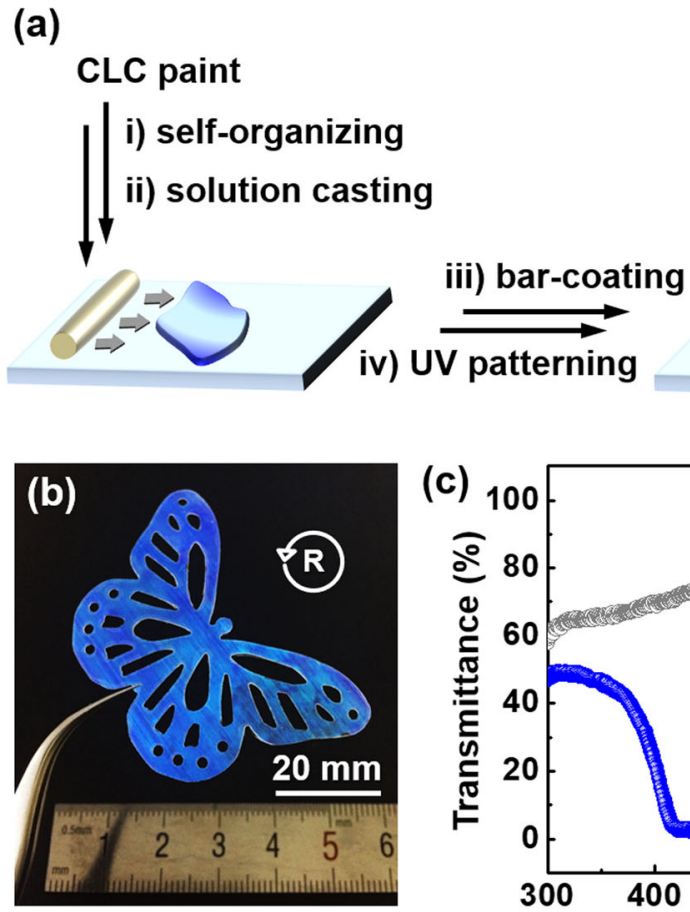

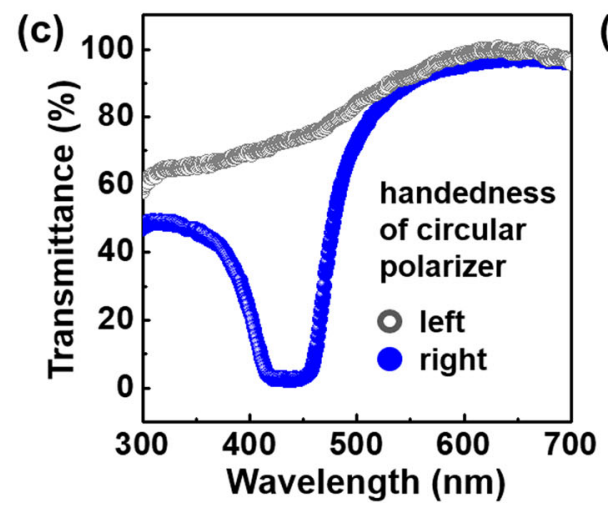

[ 1D Chirophotonic Crystal ]

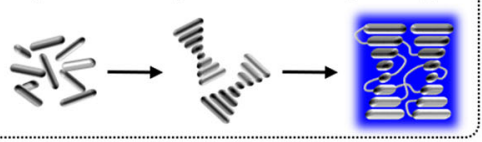

v) etching

vi) peeling

(d)

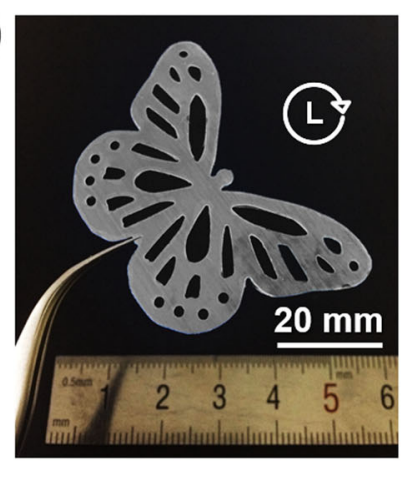

Fig. 3 Photomasking can enable the fabrication of spatially distributed objects, such as a butterfly (a). Here the butterfly is viewed under RH CPL (b). The transmission of the object under RH and LH CPL is quantified (c). The butterfly, when imaged with LH CPL, is optically transparent (d)

transmittance area of the FTIR peak at $810 \mathrm{~cm}^{-1}$, which is the out-of-plane bending vibration peak of the acrylate group $^{34}$. Figure $2 \mathrm{e}$ shows that the conversion is $81 \%$.

After the copolymerization of the chiral and achiral RMs, the CLC films are stable to chemical attack. The chemical stability of the CLC paints is first tested by dropping various solvents on them. The helical nanostructures of the CLC paints are damaged by the attack of organic solvents. For example, the CLC paints are fully dissolved and completely lose their reflection colors when exposed to chloroform. The ordered structure is disrupted by the penetration of the solvent molecules. However, the CLC films can withstand common organic solvents, as shown by the summarized results in Figure S6 in the Supporting Information. Based on the chemical stability tests, it is concluded that the construction of the crosslinked helical nanostructure is an effective method for obtaining robust photonic materials. Furthermore, it should be noted that it is possible to fabricate a patterned optical object if a photomask is applied ${ }^{35}$. Accordingly, washing the CLC films with chloroform safely removes only the unpolymerized regions. This result is illustrated in Fig. 3a. The butterfly-shaped object is free-standing (Fig. 3b). Due to the properties of the cholesteric mesophase, it retains the blue reflection and the notch at approximately $435 \mathrm{~nm}$ when probed with right-handed $(\mathrm{RH})$ circularly polarized light (CPL). However, left- handed (LH) CPL is transmitted, and no reflection color is observed (Fig. 3c) ${ }^{36}$. The butterfly-shaped object is optically clear under irradiation with LH CPL. This optical property is further characterized in Fig. 3d.

Due to the ease of processability derived from the composition used here, flexible broadband mirrors that simultaneously reflect all visible light from blue to green to red can be fabricated by the sequential processes of repetitive casting, coating, and photocuring. The sequential layering of the photonic element is illustrated in Fig. 4a. First, the CLC paint with $12 \mathrm{wt} \% \mathrm{cRM}$ is cast on a glass substrate $(10 \mu \mathrm{m}$ thickness) by a bar-coating process and then photopolymerized by UV light irradiation. As expected, the first layer (denoted $F_{1}$ ) exhibits the blue reflection color. The second layer with the green reflection is directly cast on the $F_{1}$ layer and photopolymerized. To produce $F_{3}$, a third layer with $8 \mathrm{wt} \% \mathrm{cRM}$ is prepared on the $F_{2}$ layer using the same procedure. Figure $4 \mathrm{~b}$ illustrates the stepwise change in the optical properties as the photonic element is constructed. Ultimately, a broad bandwidth $(\Delta \lambda=390 \mathrm{~nm})$ reflection spectrum, which is three times that of the $F_{1}$ layer and covers the entire visible wavelength range, is achieved for $\mathrm{F}_{3}$ with RH CPL. The reflection spectrum of the $F_{2}$ layer is only approximately twice that of the $\mathrm{F}_{1}$ layer. The thermal stability of $\mathrm{F}_{3}$ is evaluated by TGA. The CLC films are thermally stable at approximately $300^{\circ} \mathrm{C}$ and start to degrade above 


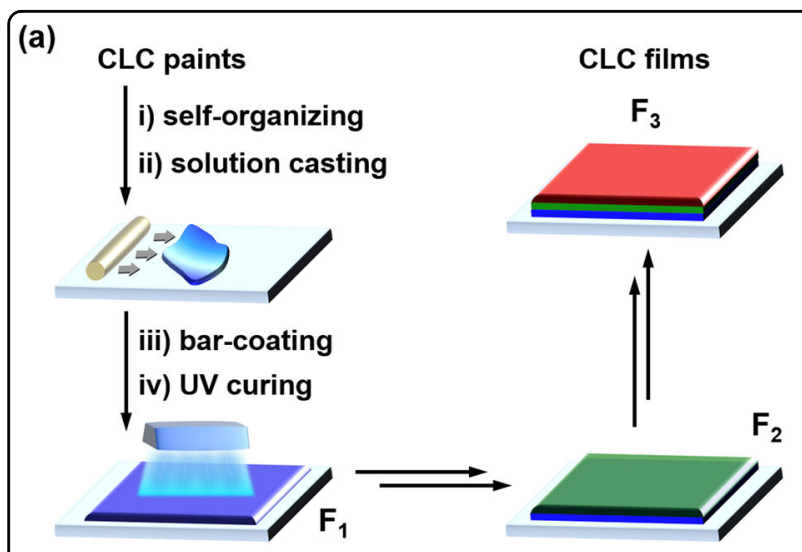

(b)

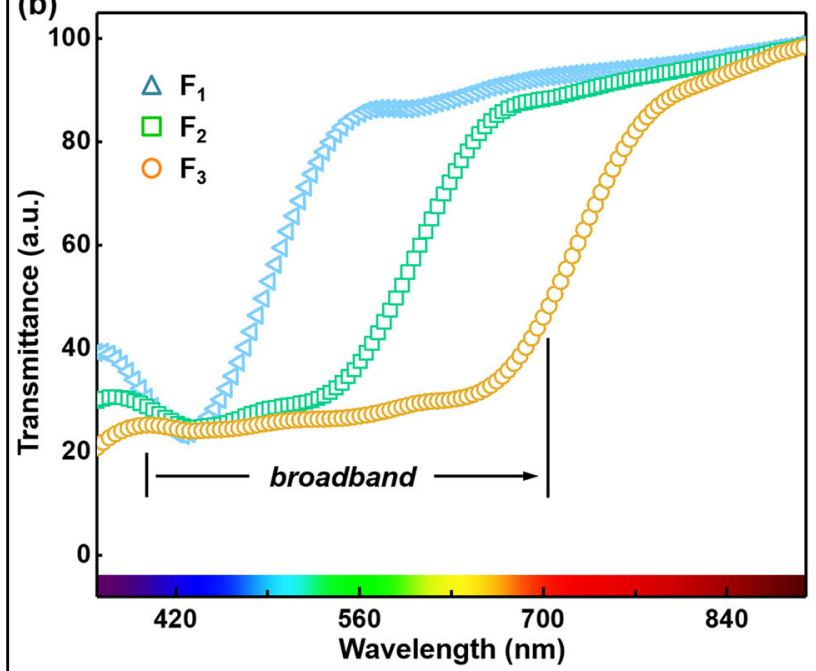

Fig. 4 Schematic illustration of the fabrication of a broadband mirror by sequential LBL and photopolymerization processes (a). Evolution of the transmittance spectra of the CLC films with different helical pitch distributions (b)

that temperature. The silver reflection of $\mathrm{F}_{3}$ is stable up to $30{ }^{\circ} \mathrm{C}$, as expected for a heavily crosslinked polymer network (Figure S7 in the Supporting Information). Next, the pencil-scratch method (ASTM D3363) is utilized to determine the mechanical robustness of the CLC films. Due to their liquid crystalline nature and low molecular weight, the CLC paints maintain their long-range orientational order and the molecular mobility of isotropic liquids. Therefore, they do not resist any mechanical force. However, $\mathrm{F}_{3}$ is resistant to scratching up to $\mathrm{HB}$ (Figure S8 in the Supporting Information). When the pencil hardness is increased to $2 \mathrm{H}$, the surface begins to be damaged ${ }^{37}$. It can be concluded that crosslinking the helical nanostructures is a robust method for protecting the photonic material.

The mirror-like silver reflection of $\mathrm{F}_{3}$ is illustrated in Fig. 5a. A common illustration of the cholesteric mesophase is visible behind $F_{3}$ (Fig. $5 \mathrm{~b}$ ). The microstructures of the CLC films are observed by 1D WAXD. A broad peak appears at approximately $2 \theta=20.13^{\circ}$, indicating the existence of the cholesteric mesophase (Figure S9 in the Supporting Information). The peak at $0.44 \mathrm{~nm}$ originates from the liquid-like correlation of the anisotropic molecules. The strong birefringence and oily streaked texture detected by POM further support the successful transfer of the chiral property and helical information of the CLC paints to the corresponding films (Figure S10 in the Supporting Information). To visualize the distinct helical nanostructures within the broadband mirrors, SEM is employed. As shown in Fig. $5 \mathrm{c}$, the cross-sectional SEM image of $\mathrm{F}_{3}$ has a $30 \mu \mathrm{m}$-thick layer (three stacked $10 \mu \mathrm{m}$ layers of CLCs). The lengths of $P_{\text {red }}=420 \mathrm{~nm}, P_{\text {green }}=$ $350 \mathrm{~nm}$, and $P_{\text {blue }}=290 \mathrm{~nm}$ are measured. The red, green, and blue layers of $\mathrm{F}_{3}$ have 24, 28, and 34 pitches, respectively. Note that only a single helical nanostructure constructed by the self-assembly of the CLC paints in the red, green, and blue layers of $\mathrm{F}_{3}$ is schematically illustrated in Fig. 5d.

\section{Discussion}

The Jones and Berreman method (a numerical model of light transmission and reflection in CLCs) indicates a minimum of 10 pitches for the total reflection notch $\operatorname{depth}^{38}$. Therefore, the maximum notch depth in this broadband mirror is $100 \%$ with $\mathrm{RH} \mathrm{CPL}{ }^{39}$. It is unexpected that $\mathrm{F}_{3}$ prepared by the LBL method on a laboratory scale exhibits a relatively high reflection notch depth ( $\sim 80 \%)$. When the CLC paint is coated on a glass substrate, the direction of the HA changes as a function of the temperature, viscosity, substrate, and shear rate. The fabrication conditions used for $\mathrm{F}_{3}$ (relatively slow shear rate and low viscosity of the CLC paint) can result in an HA perpendicular to the coating direction, instead of a completely planar or fingerprint texture. When the CLC paints are coated on a glass substrate, the CLC layer has an air interface, which can induce the homeotropic alignment of the top layer of the film ${ }^{40}$. The second and third layers have the same homeotropic layers and a less ordered HA normal to the film direction. To improve the alignment of the HA perpendicular to the substrate, further study of the fabrication conditions for the alignment of the CLC paints is needed.

\section{Conclusions}

To fabricate circular-polarizing reflective and flexible mirrors with a broad bandwidth, photopolymerizable CLC paints were first prepared by considering not only the intrinsic chirality but also the coating viscoelasticity. The helical pitch of the CLC paints were predictably controlled by the amount of the cRM in the mRM and dRM. The polymer-stabilized helical nanostructures exhibited good chemical, thermal and mechanical stabilities for fabricating patterned optical objects. The SEM 


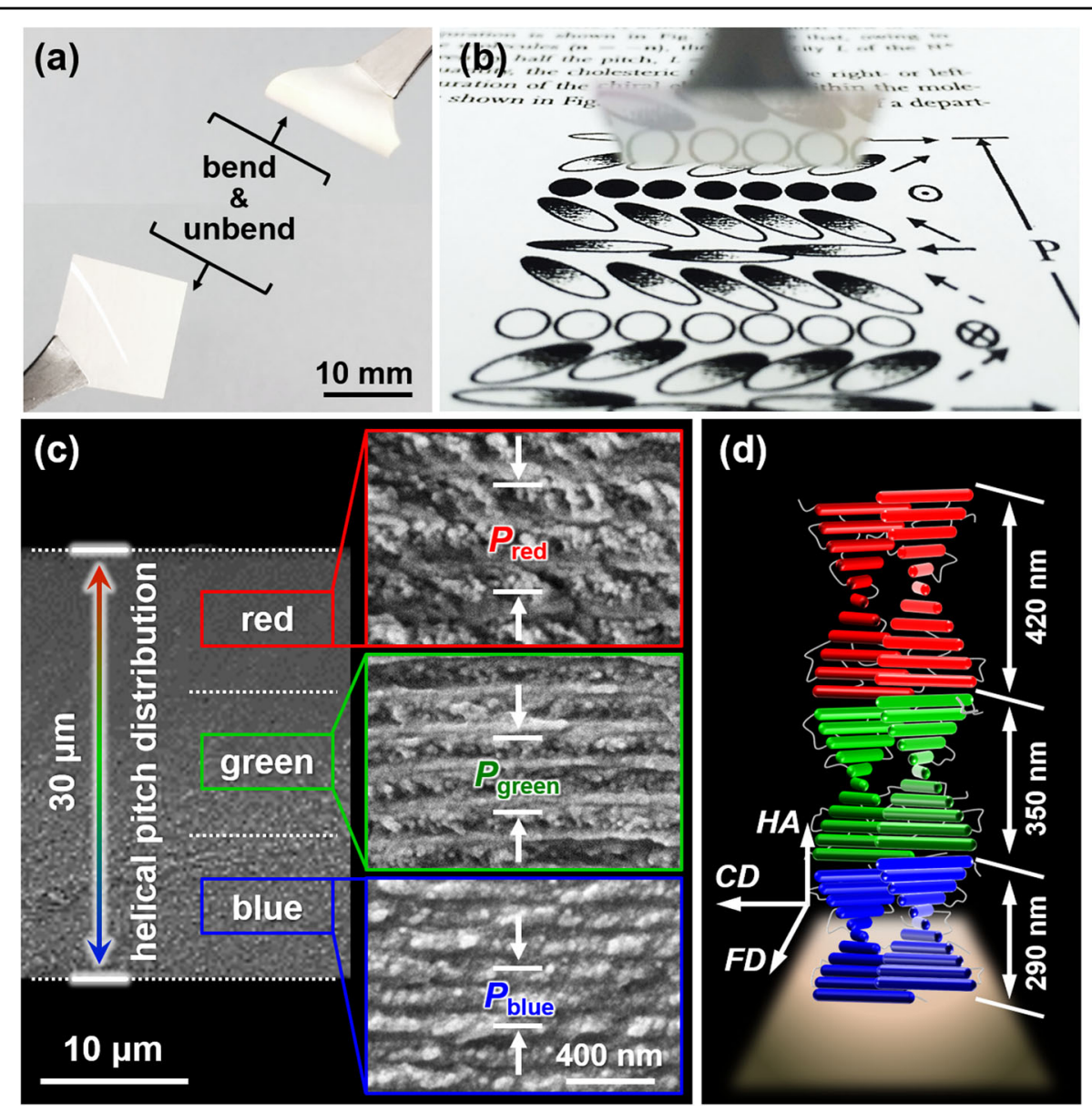

Fig. 5 Broadband mirror with a silver color and good flexibility (a). Photograph illustrating the mirror-like reflection of the $F_{3}$ sample (b). Crosssectional SEM images of the fractured $F_{3}$ sample showing the periodically stacked helical arrays with well-controlled helical pitches (c). Schematic illustration of the helical pitch distribution of the multilayer stacked nanostructure of $F_{3}(\mathbf{d})$

observations and X-ray diffraction patterns combined with optical calculations clearly showed that the sequentially LBL-coated CLC films had finely tuned helical pitches that reflected light over the entire visible wavelength range. The continuous LBL coating and subsequent in situ photopolymerization described here provides a simple solution for manufacturing large-area platforms and full-color elements at low cost.

\section{Acknowledgements}

This work was supported by the Basic Research Laboratory (2015042417, Korea) and Air Force Office of Scientific Research (FA2386-17-1-4078, USA). D.Y.K. appreciates the support of the Basic Science Research Program and CBNU Fellowship Program.

\section{Author details}

'Department of Materials Science and Engineering, Massachusetts Institute of Technology, Cambridge, MA 02139, USA. '2US Air Force Research Laboratory, Wright-Patterson Air Force Base, Dayton, $\mathrm{OH} 45433$, USA. ${ }^{3}$ Department of Chemical and Biological Engineering, University of Colorado Boulder, Boulder, CO 80309, USA. ${ }^{4}$ Department of Polymer-Nano Science and Technology, Chonbuk National University, Jeonbuk 54896, Korea
Conflict of interest

The authors declare that they have no conflict of interest.

\section{Publisher's note}

Springer Nature remains neutral with regard to jurisdictional claims in published maps and institutional affiliations.

Supplementary information is available for this paper at https://doi.org/ 10.1038/s41427-018-0096-4.

Received: 17 June 2018 Revised: 28 August 2018 Accepted: 10 September 2018.

Published online: 9 November 2018

\section{References}

1. Bisoyi, H. K. \& Li, Q. Light-directing chiral liquid crystal nanostructures: from 1d to 3d. Acc. Chem. Res. 47, 3184-3195 (2014).

2. Mitov, M. Cholesteric liquid crystals with a broad light reflection band. Adv Mater. 24, 6260-6276 (2014).

3. Matranga, A. et al. Biomimetic reflectors fabricated using self-organising, selfaligning liquid crystal polymers. Adv. Mater. 25, 520-523 (2013).

4. Binet, C., Mitov, M. \& Boudet, A. Bragg reflections in cholesteric liquid crystals; from selectivity to broadening and reciprocally. Mol. Cryst. Liq. Cryst. 339, $111-123$ (2000). 
5. Sveinbjörnsson, B. R. et al. Rapid self-assembly of brush block copolymers to photonic crystals. Proc. Natl Acad. Sci. USA 4, 14332-14336 (2012)

6. Jeong, K-U. et al. Colour-tunable spiral photonic actuators. J. Mater. Chem. 19, 1956-1959 (2009)

7. Bisoyi, H. K, Bunning, T. J. \& Li, Q. Stimuli-driven control of the helical axis of self-organized soft helical superstructures. Adv. Mater. 30, 1706512-1706546 (2018).

8. Matsui, T., Ozaki, R., Funamoto, K., Ozaki, M. \& Yoshino, K. Flexible mirrorless laser based on a free-standing film of photopolymerized cholesteric liquid crystal. Appl. Phys. Lett. 81, 3741-3743 (2002).

9. Li, Y. et al. Flexible cholesteric films with super-reflectivity and high stability based on a multi-layer helical structure. J. Mater. Chem. C 5, 10828-10833 (2017).

10. Bitar, R., Agez, G. \& Mitov, M. Cholesteric liquid crystal self-organization of gold nanoparticles. Soft Matter 7, 8198-8206 (2011).

11. Jeong, S. M. et al. Optical cavity with a double-layered cholesteric liquid crystal mirror and its prospective application to solid state laser. Appl. Phys. Lett. 89, 24116-24118 (2006)

12. Mitov, M., Binet, C., Boudet, A. \& Bourgerette, C. Glassy cholesteric broadband reflectors with a pitch gradient: Material design, optical properties and microstructure. Mol. Cryst. Liq. Cryst. 358, 209-223 (2001).

13. Kim, D.-Y. et al. Remote-controllable molecular knob in the mesomorphic helical superstructures. Adv. Funct. Mater. 26, 4242-4251 (2016).

14. Mitov, M. \& Dessaud, N. Cholesteric liquid crystalline materials reflecting more than 50\% of unpolarized incident light intensity. Liq. Cryst. 34, 183-193 (2007).

15. Makow, D. M. \& Sanders, C. L. Additive colour properties and colour gamut of cholesteric liquid crystals. Nature 276, 48-50 (1978).

16. Sixou, P., Nourry, J., Guillard, H. \& Gautier, C. Colour rendering of polymer network cholesteric liquid crystal: model and experimental results. Mol. Cryst. Liq. Cryst. 365, 467-479 (2001).

17. Mitov, M., Boudet, A. \& Sopena, P. From selective to wide-band light reflection: a simple thermal diffusion in a glassy cholesteric liquid crystal. Eur. Phys. J. B 8, 327-330 (1999).

18. Zografopoulos, D. C., Kriezid, E. E., Mitov, M. \& Binet, C. Theoretical and experimental optical studies of cholesteric liquid crystal films with thermally induced pitch gradients. Phys. Rev. E 73, 061701-061709 (2006).

19. Chen, G. et al. Photoinduced hyper-reflective laminated liquid crystal film with simultaneous multicolor reflection. ACS Appl. Mater. Interfaces 6, 1380-1384 (2014).

20. Lee, K. M. et al. Color-tunable mirrors based on electrically regulated bandwidth broadening in polymer-stabilized cholesteric liquid crystals. ACS Photonics 1, 1033-1041 (2014).

21. Li, Y., Xue, C., Wang, M., Urbas, A. \& Li, Q. Photodynamic chiral molecular switches with thermal stability: from reflection wavelength tuning to handedness inversion of self-organized helical superstructures. Angew. Chem. Int. Ed. 52, 13703-13707 (2013)
22. Chen, J. et al. Self-healing responsive chiral photonic films for sensing and encoding. J. Mater. Chem. C 6, 7767-7775 (2018).

23. Khan, M. K. et al. Flexible mesoporous photonic resins with tunable chira nematic structures. Angew. Chem. Int. Ed. 52, 8921-8924 (2013).

24. Ryabchun, A., Sakhno, O., Stumpe, J. \& Bobrovsky, A. Full-polymer cholesteric composites for transmission and reflection holographic gratings. Adv. Opt. Mater. 5, 1700314-1700323 (2017).

25. Guillard, H. \& Sixou, P. Active broadband polymer stabilized liquid crystals. Liq. Cryst. 28, 933-944 (2001).

26. Broer, D. J., Mol, G. N., Haaren, J. A. M. M. V. \& Lub, J. Photo-induced diffusion in polymerizing chiral-nematic media. Adv. Mater. 11, 573-578 (1999).

27. Hikmet, R. A. M. \& Kemperman, H. Electrically switchable mirrors and optical components made from liquid-crystal gels. Nature 392, 476-479 (1998).

28. Chen, X. et al. Broadband reflection of polymer-stabilized chiral nematic liquid crystals induced by a chiral azobenzene compound. Chem. Commun. $\mathbf{5 0}$ 691-694 (2014)

29. Kim, D.-Y. et al. Free-standing and circular-polarizing chirophotonic crystal reflectors: photopolymerization of helical nanostructures. ACS Nano $\mathbf{1 0}$ 9570-9576 (2016)

30. Baliyan, V. K. Lee, S. H. \& Kang, S.-W. Optically and spatially templated polymer architectures formed by photopolymerization of reactive mesogens in periodically deformed liquid crystals. NPG Asia Mater. 9, 429-436 (2017).

31. Lee, B.-Y. \& Lee, J.H. Printable flexible cholesteric capsule display with a fine resolution of RGB subpixels. Curr. Appl. Phys. 11, 1389-1393 (2011).

32. Bayer, I. S. et al. Thermal alternating polymer nanocomposite (TAPNC) coating designed to prevent aerodynamic insect fouling. Sci. Rep. 6, 38459-38471 (2016).

33. Herzer, N. et al. Printable optical sensors based on $\mathrm{H}$-bonded supramolecular cholesteric liquid crystal networks. J. Am. Chem. Soc. 134, 7608-7611 (2012).

34. Lee, K. M. et al. Initiatorless photopolymerization of liquid crystal monomers ACS Appl. Mater. Interfaces 8, 28040-28046 (2016).

35. Lub, J., Nijssen, W. P. M., Wegh, R. T., Vogels, J. P. A. \& Ferrer, A. Synthesis and properties of photoisomerizable derivatives of isosorbide and their use in cholesteric filters. Adv. Funct. Mater. 15, 1961-1972 (2005).

36. Wang, H. et al. Photochemically and thermally driven full-color reflection in a self-organized helical superstructure enabled by a halogen-bonded chiral molecular switch. Angew. Chem. Int. Ed. 57, 1627-1631 (2018).

37. Park, S.-K. et al. Polymer-stabilized chromonic liquid-crystalline polarizer. Adv. Funct. Mater. 21, 2129-2139 (2011).

38. $\mathrm{Xu}, \mathrm{M} ., \mathrm{Xu}, \mathrm{F} . \&$ Yang, D. K. Effects of cell structure on the reflection of cholesteric liquid crystal display. J. Appl. Phys. 83, 1938-1944 (1998).

39. McConney, M. E. et al. Thermally induced, multicolored hyper-reflective cholesteric liquid crystals. Adv. Mater. 23, 1453-1457 (2011).

40. Cha, Y. J. et al. Orthogonal liquid crystal alignment layer: templating speeddependent orientation of chromonic liquid crystals. ACS Appl. Mater. Interfaces 9, 18355-18361 (2017). 